\title{
Investigating multi-polarization GPR wave transmission through thin layers: Implications for vertical fracture characterization
}

\author{
Georgios P. Tsoflias ${ }^{1}$ and Anthony Hoch ${ }^{1}$ \\ Received 7 August 2006; revised 12 September 2006; accepted 15 September 2006; published 18 October 2006.
}

[1] We investigate the controls governing the response of ground-penetrating radar (GPR) wave transmission through thin layers in order to explore the use of variable polarization GPR signals for remote characterization of fracture aperture and fluid-fill. We employ an experimental setting that provides controlled observations of the effects of thin-layer properties to the transmitted GPR wavefield. GPR signals of variable polarization, variable angle of incidence, and variable frequency are transmitted through an air- and water-filled layer of variable thickness. We observe that at high angles of incidence, variable polarization GPR signals display characteristic and quantifiable phase and amplitude responses that are related to thin-layer properties. The GPR data are in agreement to analytical solutions of plane-wave oblique-incidence transmission through layered media. We conclude that multi-polarization GPR observations can be exploited to determine fracture properties. This work has implications in the remote determination of fractured formation anisotropic properties, such as fluid-flow. Citation: Tsoflias, G. P., and A. Hoch (2006), Investigating multi-polarization GPR wave transmission through thin layers: Implications for vertical fracture characterization, Geophys. Res. Lett., 33, L20401, doi:10.1029/2006GL027788.

\section{Introduction}

[2] We present a study of the phase and amplitude changes that occur to ground-penetrating radar (GPR) waves as they transmit through a thin layer, and we relate those changes to the properties of the layer. In geologic environments, fractures form thin layers with sub-wavelength apertures that are commonly targeted by GPR surveys. Remote determination of fracture properties, such as fracture aperture and fluid content, are of particular interest to the study of subsurface fluid-flow properties and for predicting the transport of contaminants. For example laminar flow in a fracture is proportional to the third power of fracture aperture [Snow, 1969; Lamb, 1932]. Therefore, in addition to employing GPR for locating fractures and imaging fracture planes [e.g., Grasmueck, 1996; Grasmueck et al., 2005; Seol et al., 2001], remote determination of fracture aperture and fill is essential for hydrogeologic studies.

[3] Recent studies have employed the amplitude characteristics of reflected GPR signals from horizontal and subhorizontal fractures to determine fracture aperture variability [Grégoire et al., 2003; Grégoire and Hollender, 2004] and to

\footnotetext{
${ }^{1}$ Department of Geology, University of Kansas, Lawrence, Kansas, USA.
}

Copyright 2006 by the American Geophysical Union. 0094-8276/06/2006GL027788 monitor fracture water content variability [Tsoflias et al., 2001] and saline tracer flow along fractures [Talley et al., 2005]. Day-Lewis et al. [2003] employed time-lapse borehole radar to monitor saline tracer flow through fractured crystalline rocks. These studies showed that single offset, near-normal incidence, constant polarization GPR data yield amplitude responses that relate to fracture properties or changes of fracture properties, such as fluid salinity and saturation. However, the remote determination of fracture properties from GPR signal responses remains a challenging task due to the non-uniqueness of reflection amplitude responses and the difficulty of imaging vertical, nonreflecting fractures.

[4] Depending on radar survey acquisition geometry (i.e., surface reflection, borehole tomography or transillumination) and fracture plane orientation (vertical, horizontal or subhorizontal), impinging GPR waves interfere with fractures at varying incidence and polarization angles. Therefore, it is important to consider these additional radar wavefield variables in using GPR in the study of fractures. Tsoflias et al. [2004] recognized that bi-static, surface GPR reflection acquisition geometry results in high-angle of incidence transmission across a vertical fracture located between the transmitting and receiving antennas, and proposed multipolarization GPR acquisition for detection of the vertical fracture. Based on the phase difference between orthogonally polarized GPR data pairs, the location and azimuth of vertical fractures were identified in a fractured carbonate aquifer [Tsoflias et al., 2004].

[5] The work presented here extends previous work by providing experimental and theoretical investigation of the controls of GPR wave transmission though a thin layer. Understanding the interaction of radar waves with fractures (or thin layers) can aid in the remote prediction of fracture properties. We employed controlled experiments to investigate the dependence of EM wavefield properties, such as polarization, angle of incidence and frequency, to layer properties such as thickness and fluid content. We compared the experimental data to analytical solutions of plane wave oblique incidence transmission through planar layered media. Our experimental and analytical observations show good agreement and reveal characteristic EM wave amplitude and phase responses to transmission through thin layers that are related to layer properties. Therefore, we conclude that multipolarization GPR surveys can be used to quantify aperture and fill of fractures. We place particular emphasis on changes occurring to the phase of the radar signals. GPR investigations have overwhelmingly emphasized the amplitude response of radar waves. We show that phase is an important signal attribute that should be considered in studies of subsurface properties. This study advances the understanding of the relationships between EM wave polarization properties 


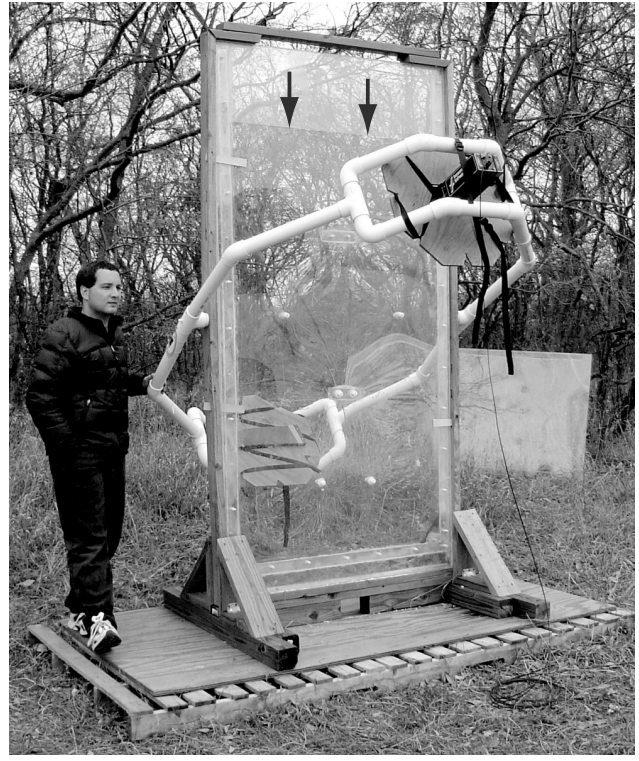

Figure 1. Experimental setting of a simulated fresh-water filled, $4.8 \mathrm{~mm}$ aperture thin layer. $200 \mathrm{MHz}$ radar signals parallelly polarized to the thin layer, E-pol, are transmitted at variable angles of incidence $\left(0^{\circ}-70^{\circ}\right)$ controlled by the pivoting arm. Rotating both antennas by $90^{\circ}$ results in perpendicularly polarized signals impinging to the plane of the thin layer, H-pol. The water level in the simulated fracture is marked by arrows.

and thin, sub-wavelength thickness zones of subsurface anisotropy.

\section{Methods}

[6] In order to test the effects of transmission through a thin layer on GPR wavefields, an experimental setup was constructed that allowed controlled observations. The results of the controlled tests were then compared to analytical solutions of EM wave transmission through multiple interfaces.

\subsection{Experimental Setting}

[7] The controlled experiments involved transmission of varying polarization, varying frequency, and varying angle of incidence GPR signals through a vertical thin layer. In order to study the effects of layer thickness and fluid content on GPR wavefield phase and amplitude, the media surrounding the thin layer had to be homogenous and the model had to be sufficiently large to yield far-field observations at the $100 \mathrm{MHz}$ to $1 \mathrm{GHz}$ frequency range commonly used in GPR surveys. Furthermore, the model had to allow consistent positioning of antennas (constant separation and orientation) of differing physical dimensions (varying frequencies) over a wide range of angles of incidence to the thin layer. Therefore, we constructed the model consisting of layers of air, allowing maximum control of antenna positioning, and polycarbonate, a medium nearly transparent to radar waves (relative permittivity $\varepsilon_{r}=3$, electrical conductivity $\sigma=1 \mathrm{mS} / \mathrm{m}$ ). The vertical thin layer was simulated by the space between two $1.22 \times$ $2.44 \mathrm{~m}(4 \times 8$ foot $), 12.7 \mathrm{~mm}(0.5$ inch $)$ thick sheets of polycarbonate. The space between the polycarbonate sheets was set to $1.6 \mathrm{~mm}(1 / 16 \mathrm{inch})$ and $4.8 \mathrm{~mm}$ (3/16 inch) to simulate different layer thicknesses. Acrylic bolts and polycarbonate spacers were used to ensure uniform gap between the large polycarbonate sheets, although some limited bulging occurred from the weight of the water when simulating the $4.8 \mathrm{~mm}$ aperture. The space between the sheets was filled with air $\left(\varepsilon_{r}=1, \sigma=0 \mathrm{mS} / \mathrm{m}\right)$, fresh water $\left(\varepsilon_{r}=80, \sigma=50 \mathrm{mS} / \mathrm{m}\right)$ or saline water $\left(\varepsilon_{r}=80\right.$, $\sigma=6550 \mathrm{mS} / \mathrm{m}$ ). The GPR antennas were mounted on a pivoting arm facing each other and they were separated by a constant offset of $2 \mathrm{~m}$ throughout the experiment (Figure 1). The radar signal was transmitted through the simulated thin layer at angles of incidence ranging from $0^{\circ}$ to $70^{\circ}$, at $5^{\circ}$ angle increments, and 10 traces were acquired at each angle of incidence. Data were recorded at sampling intervals of $0.01 \mathrm{~ns}, 0.02 \mathrm{~ns}$ and $0.05 \mathrm{~ns}$. Data were collected using a PulseEKKO 100 GPR system at frequencies of 100 and $200 \mathrm{MHz}$ and a PulseEKKO 1000 system at frequencies of 225, 450 and $900 \mathrm{MHz}$. These GPR systems use dipole antennas, which generate linearly polarized EM fields with the electric field oriented along the long axis of the antenna. Making multi-polarization observations involves rotating the orientation of the antennas relative to the polycarbonate sheets. For the entire range of angles of
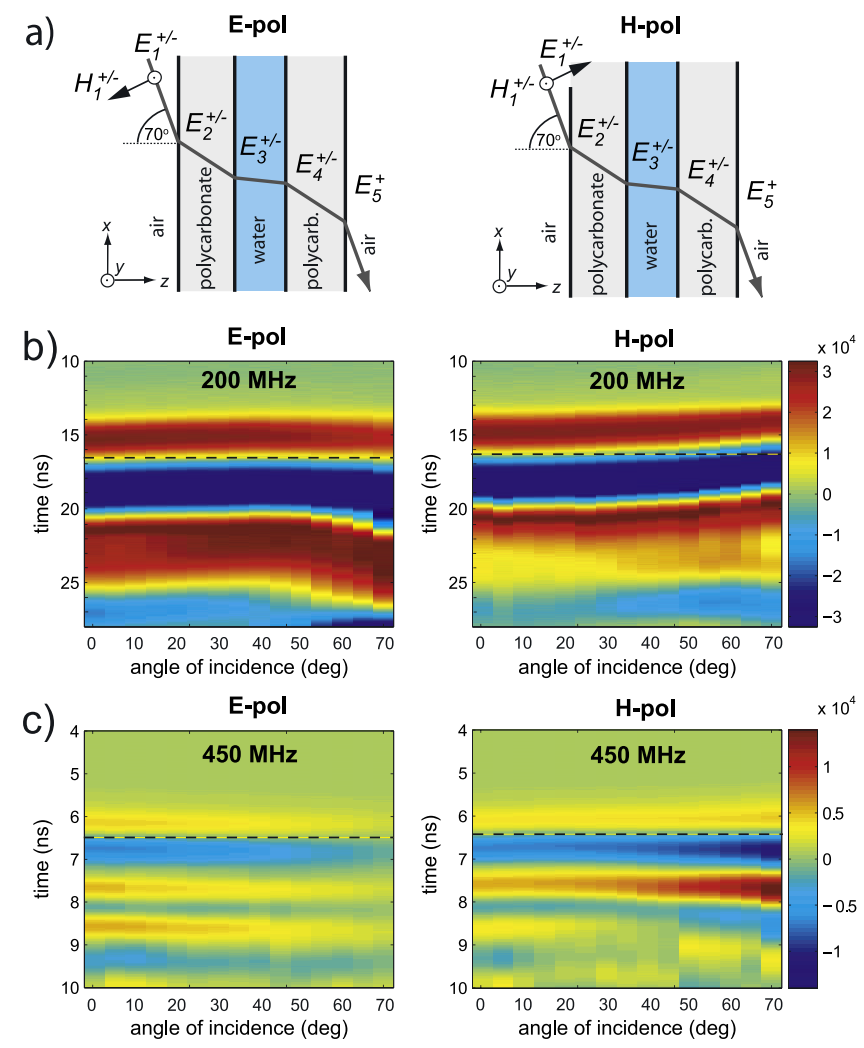

Figure 2. (a) EM wave ray tracing schematics of the experimental setup at $70^{\circ}$ angle of incidence for E-pol and H-pol. $E_{\#}^{+-}$denotes the total electric field in regions \#1 through \#4 with the incident wave propagating in $+z$ direction and the reflected wave propagating in opposite -z direction. $E_{5}^{+}$is the transmitted field in half-space region 5 . E-pol and H-pol (b) $200 \mathrm{MHz}$ and (c) $450 \mathrm{MHz}$ GPR data transmitted at $5^{\circ}$ angle of incidence increments across a $4.8 \mathrm{~mm}$ thick fresh water filled layer. Horizontal dashed lines are drawn at the zero-crossing of the normal incidence direct arrival for time shift reference. 

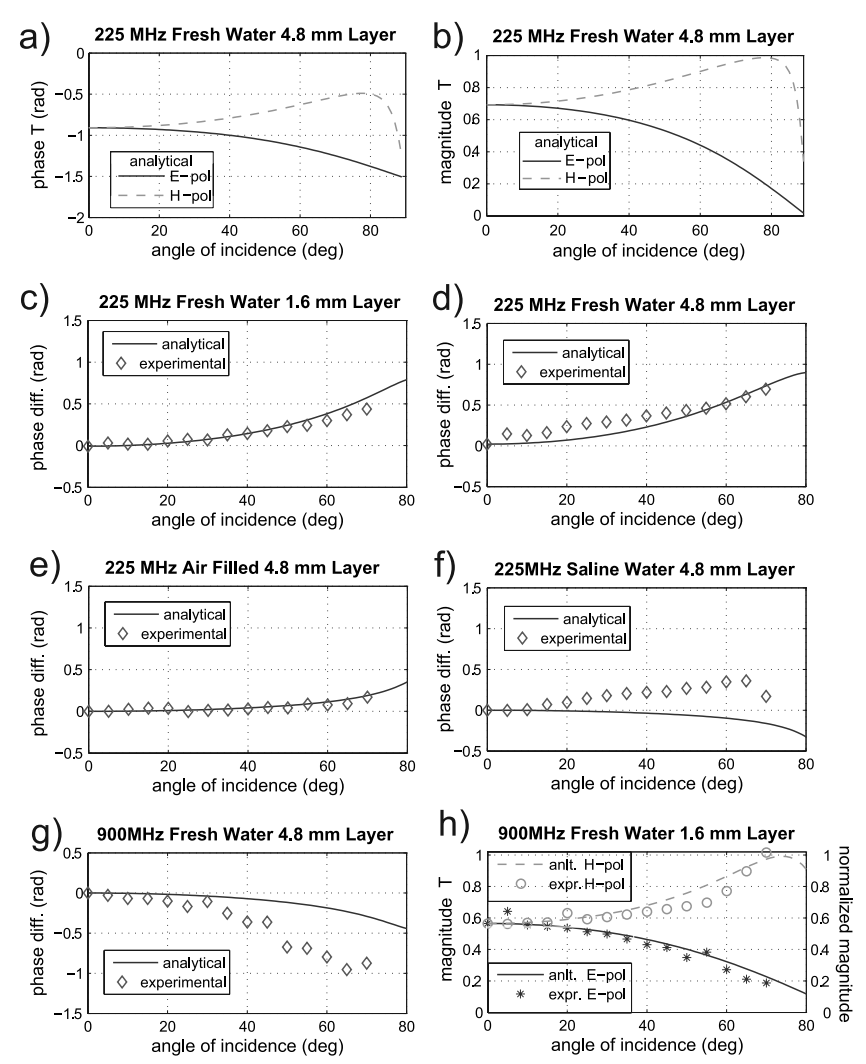

Figure 3. Analytical solutions of (a) phase and (b) magnitude of the net four-interface E-pol $\left(T_{E}\right)$ and H-pol $\left(T_{H}\right)$ transmission coefficients for a $4.8 \mathrm{~mm}$ thick, fresh-water filled layer at $225 \mathrm{MHz}$ signal frequency. (c-h) Comparison of analytical solutions and experimental data for variable EM wave and thin layer properties. Refer to text for detailed description.

incidence, $0^{\circ}$ polarization transmission (E-pol) relative to the thin layer is achieved when the long axis of the antenna is oriented horizontally as shown in Figure 1. Ninety degree polarization transmission ( $\mathrm{H}-\mathrm{pol})$ is achieved when the antennas are rotated $90^{\circ}$, oriented vertically. Figure 2a shows a ray-trace schematic of the two EM wave polarization states, E-pol and H-pol, and Figures $2 \mathrm{~b}$ and $2 \mathrm{c}$ display data collected with the experimental setup.

\subsection{Analytical Solutions}

[8] We employed the recursive method used in EM plane wave studies, which considers field continuity and matching of the impedances across boundaries [Balanis, 1989] to analytically compute the response of the experimental setup described in the previous section. We computed oblique incidence, variable frequency, $0^{\circ}$ polarization relative to the thin layer (E-pol) and $90^{\circ}$ polarization (H-pol) complex transmission and reflection coefficients, and electric and magnetic fields, through a five-layer lossy model. The analytic model is shown in Figure 2a consisting of: [open air] - [12.7 $\mathrm{mm}$ polycarbonate $]-[1.6$ and $4.8 \mathrm{~mm}$ thick layer filled alternately with air, fresh and saline water] [12.7 $\mathrm{mm}$ polycarbonate] - [open air]. Analytical computations of the net (or total effect) four-interface transmission coefficient magnitude and phase are evaluated as the ratio of the electric field transmitted in region five to the incident field in region one. The complex E-pol and H-pol net transmission coefficients, $T_{E}$ and $T_{H}$ respectively, are given by

$$
T_{E}=\frac{E_{5 y}^{+}}{E_{1 y}^{+}} \quad \text { and } \quad T_{H}=\frac{E_{5 x}^{+}}{E_{1 x}^{+}},
$$

where " $y$ " and " $x$ " subscripts indicate the direction of electric field components tangential to the interfaces. Figures $3 \mathrm{a}$ and $3 \mathrm{~b}$ display analytical computations of net $T_{E}$ and $T_{H}$ phase and magnitude as a function of angle of incidence.

\section{Results}

\subsection{Controlled Experiment Results}

[9] The schematic in Figure 2a ray-traces a GPR wave impinging at $70^{\circ}$ and transmitting through the four-interface model at E-pol and H-pol polarization states. The raypath is described by Snell's law, which is independent of wave polarization. Thus, for the GPR wave impinging at $70^{\circ}$, both E-pol and H-pol transmitted waves travel the same path length at the same velocity of propagation and therefore they should transmit across the four interfaces at the same traveltime. Figure 2b displays E-pol and H-pol, $200 \mathrm{MHz}$ GPR signals transmitted through a $4.8 \mathrm{~mm}$ thick fresh water-filled layer. At angles of incidence greater than $40^{\circ}$ both polarization data sets display apparent time shifts of opposite sense that increase as the angle of incidence increases. At high angles of incidence, parallelly polarized signals to the fracture plane (E-pol) appear delayed in time relative to lower angle of incidence transmission, whereas perpendicularly polarized signals appear earlier in time (H-pol). At $70^{\circ}$ angle of incidence the direct arrival time difference between H-pol and E-pol signals is $0.9 \mathrm{~ns}$, despite the fact that both sets of radar waves traveled the same path through the same media. The travelpath difference between normal incidence and $70^{\circ}$ oblique incidence transmission through the model with a middle layer filled with water (layer 3 ) is estimated to add $0.03 \mathrm{~ns}$ of traveltime to both polarization signals. Figure $2 \mathrm{c}$ shows data acquired with the same physical model and $450 \mathrm{MHz}$ frequency radar signals. The arrival time difference between orthogonal polarization data has decreased significantly compared to the $200 \mathrm{MHz}$ data of Figure $2 \mathrm{~b}$.

[10] The amplitude response of orthogonal polarization data also displays characteristic and detectable differences as a function of angle of incidence. Figure $2 \mathrm{c}$ shows that H-pol net transmission amplitude increases with increasing angle of incidence, whereas E-pol net transmission amplitude decreases. Amplitudes of the $200 \mathrm{MHz}$ signals (Figure 2b) are not examined because of received signal amplitude saturation caused by the lower frequency high power transmitter.

\subsection{Analytical Results}

[11] Analytical computation of the phase of orthogonal polarization net transmission coefficients for the experimental setting of Figure 1 are shown in Figure $3 \mathrm{a}$ and indicate a relative phase lead for $\mathrm{H}-$ pol and a phase lag for E-pol. These analytical trends are in agreement to the observed data time shifts in Figure $2 \mathrm{~b}$. Therefore, the apparent time shifts observed between the two orthogonal polarization signals at high angle of incidence transmission through the thin layer are phase shifts relating to the characteristics of the net 
transmission coefficient. A direct comparison of analytical and experimental results is presented in the next section.

[12] Figure $3 \mathrm{~b}$ indicates that at oblique angles of incidence the magnitude of the H-pol net transmission coefficient is greater than the magnitude of the E-pol transmission. These analytical computations show trends that are in agreement to the amplitude response of the experimental observations described above (Figure 2c). H-pol net transmission coefficient magnitude is shown to reach a maximum value of 1.0 at $78^{\circ}$ angle of incidence (Figure $3 \mathrm{~b}$ ). It should be noted that the phenomenon of total transmission known as Brewster's angle only occurs for EM waves polarized parallel to the incidence plane, i.e., H-pol in this study. For a simplified model with a single interface of polycarbonate and water, Brewster's angle of total transmission occurs at an incidence angle of $79^{\circ}$ which is in good agreement to the analytical result for the entire four-interface model.

\subsection{Comparison of Experimental and Analytical Results}

[13] Arrival time differences between H-pol and E-pol waves of the experimental data were converted to phase differences in radians and were compared to the corresponding analytical model phase differences between H-pol and E-pol net transmission coefficients (Figures $3 c-3 g$ ). Experimental GPR data amplitudes of orthogonal polarizations were normalized to the magnitude of the corresponding normal incidence $\left(0^{\circ}\right)$ analytical H-pol and E-pol net transmission coefficients and compared to the magnitudes of the transmission coefficients as a function of angle of incidence (Figure $3 \mathrm{~h}$ ). Both phase and amplitude values of the experimental data are in general agreement with the analytically computed transmission coefficients.

[14] H-pol and E-pol $225 \mathrm{MHz}$ frequency wave phase differences are shown for varying layer thickness (Figure 3c vs. Figure 3d), varying fluid content (Figure 3d vs. Figure 3e), and varying fluid salinity (Figure $3 d$ vs. Figure $3 f$ ). Experimental data and analytical modeling indicate that water filling the $4.8 \mathrm{~mm}$ layer introduces a greater H-pol phase lead compared to an air-filled layer. Introduction of saline water $(\sigma=6550 \mathrm{mS} / \mathrm{m})$ to the $4.8 \mathrm{~mm}$ layer yields experimental data that display decreased $\mathrm{H}$-pol phase lead relative to fresh water fill, and a phase lead trend reversal at $70^{\circ}$ angle of incidence. The saline water analytical model also shows decreased H-pol phase lead compared to fresh water fill. The saline water analytical model actually indicates a phase lead reversal, i.e., E-pol phase lead, which is not observed in the experimental data except perhaps at the $70^{\circ}$ incidence angle where the phase lead trend reverses. It should be noted that the analytical models do not account for propagation effects that are more complex in electrically conductive environments and can introduce differences between experimental and modeled data. With respect to layer thickness variability, reduced magnitude phase changes are observed compared to fluid-fill changes, but still good agreement is shown between analytical and experimental data. A $4.8 \mathrm{~mm}$ water-filled layer is shown to result in a slightly higher H-pol phase lead compared to a $1.6 \mathrm{~mm}$ layer. For high angle transmission (i.e., $70^{\circ}$ incidence angle) through a $4.8 \mathrm{~mm}$ water-filled layer, increasing signal frequency decreases $\mathrm{H}$-pol phase lead from $0.9 \mathrm{~ns}$ (or $0.8 \mathrm{rad}$ ) at $200 \mathrm{MHz}$ (Figure 2b) to $0.7 \mathrm{rad}$ at $225 \mathrm{MHZ}$ (Figure 3d), to $0.15 \mathrm{~ns}$ (or $0.4 \mathrm{rad}$ ) at $450 \mathrm{MHz}$
(Figure 2c), to a reversal of the phase lead relationship with E-pol leading at $900 \mathrm{MHz}$ frequency.

[15] The amplitude responses of orthogonally polarized signals of varying frequency through varying layer thickness and fluid content yield consistent H-pol amplitudes increasing with increasing angle of incidence up to a maximum value observed at angles approaching the Brewster angle, followed by a rapid decline. E-pol amplitudes decrease with increasing angle of incidence (Figures 2c, 3b, and $3 \mathrm{~h}$ ). Increased fluid conductivity results in an overall decrease of transmitted signal amplitudes for both polarizations, with H-pol displaying higher amplitudes than E-pol with increasing angle of incidence.

\section{Discussion and Conclusions}

[16] Our investigation of the controls governing the response of ground-penetrating radar (GPR) wave high-angle of incidence transmission through thin layers shows that for a range of signal frequencies varying polarization GPR signals display characteristic and quantifiable phase and amplitude responses that are related to layer properties.

[17] The findings of this work can be summarized as follows with regard to amplitude and phase differences between orthogonally polarized signals transmitted through the four-interface model:

[18] 1. Angle of incidence: Increasing incidence angle to a thin layer results in increasing phase and amplitude differences.

[19] 2. EM Wave Polarization: H-pol signal amplitudes are consistently higher than E-pol signal amplitudes and can be used to detect the presence of a thin layer. The H-pol vs. E-pol phase lead relationship exhibits a signal wavelength (or frequency) to layer thickness dependence and therefore offers the capability to discriminate between varying thickness layers.

[20] 3. Layer thickness: For mm scale layer thickness, lower frequency GPR signals (100 and $200 \mathrm{MHz}$ ) result in greater H-pol phase lead compared to higher frequency signals $(450 \mathrm{MHz})$; increasing frequency $(900 \mathrm{MHz})$ results in reversal of the phase lead relationship. It should be noted that this observation is contrary to the common perception, derived from normal incidence reflection methods, that higher frequencies offer greater resolving power.

[21] 4. Fluid content: Water-filled layers display greater phase difference variability than air-filled layers. Highsalinity water results in decreasing H-pol phase lead and an overall decrease in transmitted signal amplitudes.

[22] It is important to note that corresponding experimental data and analytic computations showed remarkably good agreement, considering that the analytic solutions did not account for wave propagation effects such as signal dispersion, complex modes of propagation, and antenna radiation effects. The effects of these differences were minimized in the experimental setup by propagating through homogeneous low loss media such as air, polycarbonate and fresh water, and by ensuring that the antennas were kept at constant offset facing each other throughout the experiments. The influence of the polycarbonate layers can be seen by the response of the experimental set-up in air as shown in Figure 3e. Saline water experimental data resulted in the least consistent observations of polarization dependent phase differences and the least 
favorable agreement with analytical models. We attribute these inconsistencies to high electrical conductivity of the fluid affecting the transmission of GPR waves through the model.

[23] The motivation for the work presented here is characterization of fracture properties. However, the results of this study can have an impact for any GPR investigation that involves high angle transmission of radar waves through layered media. One example of such survey geometry is cross-borehole tomography where $\mathrm{H}$-pol data are transmitted through horizontally stratified geologic formations. The resulting apparent time shifts, that can be of the order of a nanosecond as shown in Figure $2 b$, will introduce errors to the tomographic inversions that rely on accurate EM wave arrival times for subsurface property estimation. Widely used ray-based inversion methods do not account for the potential of erroneous arrival times due to signal phase shifts.

[24] Although the experimental setup employed in this study does not represent a fractured geologic formation, it offered the capability to investigate the controls of radar wave transmission though thin layers and it allowed us to assess the use of analytical solutions in predicting the response of multipolarization GPR waves transmitting through thin layers. The good agreement between observed experimental data and analytical solutions suggests that radar amplitude and phase responses can be related to fracture aperture and fluid content. We conclude that multi-polarization GPR observations can be exploited to determine the properties of fractures. These results have implications in the remote characterization of anisotropic subsurface properties, such as flow through fractured formations. Ongoing research is expanding the findings of the work presented here to naturally fractured geologic formations.

[25] Acknowledgments. We would like to thank Jon Jarvis and Mike McGlashan for assistance in the field and Sally Hayden for manuscript edits.
Funding for this research was provided by The University of Kansas New Faculty General Research Fund and The American Chemical Society PRF 43401-G8. Acquisition of the GPR system was funded by the National Science Foundation EAR/IF-0345445.

\section{References}

Balanis, C. (1989), Advanced Engineering Electromagnetics, pp. 220-243, John Wiley, Hoboken, N. J.

Day-Lewis, F. D., J. W. Lane Jr., J. M. Harris, and S. M. Gorelick (2003), Time-lapse imaging of saline-tracer transport in fractured rock using difference-attenuation radar tomography, Water Resour. Res., 39(10), 1290, doi:10.1029/2002WR001722.

Grasmueck, M. (1996), 3-D ground-penetrating radar applied to fracture imaging in gneiss, Geophysics, 61, 1050-1064.

Grasmueck, M., R. Weger, and H. Horstmeyer (2005), Full resolution 3-D GPR imaging, Geophysics, 70, K12-K19.

Grégoire, C., and F. Hollender (2004), Discontinuity characterization by the inversion of the spectral content of ground-penetrating-radar (GPR) reflection-Application of the Jonscher model, Geophysics, 69, 14141424

Grégoire, C., L. Halleux, and V. Lukas (2003), GPR abilities for the detection and characterisation of open fractures in a salt mine, Near Surf. Geophys., 1, 139-147.

Lamb, H. (1932), Hydrodynamics. (Reissued by Dover, Mineola, N. Y., 1993.)

Seol, S. J., et al. (2001), Finding the strike direction of fractures using GPR, Geophys. Prospect., 49(3), 300-308.

Snow, D. T. (1969), Anisotropic permeability of fractured media, Water Resour. Res., 5, 1273-1289.

Talley, J., G. S. Baker, M. W. Becker, and N. Beyrle (2005), Four dimensional mapping of tracer channelization in subhorizontal bedrock fractures using surface ground penetrating radar, Geophys. Res. Lett., 32, L04401, doi:10.1029/2004GL021974.

Tsoflias, G. P., T. Halihan, and J. M. Sharp Jr. (2001), Monitoring pumping test response in a fractured aquifer using ground penetrating radar, Water Resour. Res., 37, 1221-1229.

Tsoflias, G. P., J. P. Van Gestel, P. Stoffa, D. Blankenship, and M. Sen (2004), Vertical fracture detection by exploiting the polarization properties of GPR signal, Geophysics, 69, 803-810.

A. Hoch and G. P. Tsoflias, Department of Geology, University of Kansas, 120 Lindley Hall, 1475 Jayhawk Blvd., Lawrence, KS 66045, USA. (tsoflias@ku.edu) 